\title{
Implementação da IoT para o Monitoramento das Variáveis Meteorológicas num AAL
}

\author{
Madalena Pereira da Silva ${ }^{1}$, Débora Cabral Nazário ${ }^{1}$, M.A.R. Dantas ${ }^{1,2}$, Alexandre \\ Leopoldo Gonçalves ${ }^{1}$, A.R. Pinto ${ }^{3}$, Guilherme Manerichi ${ }^{3}$, Bruno Vanelli ${ }^{3}$
}

${ }^{1}$ Engineering and Knowledge Management Department, ${ }^{2}$ Computer Science and Statistics Department, ${ }^{3}$ Control and Automation Engineering Department

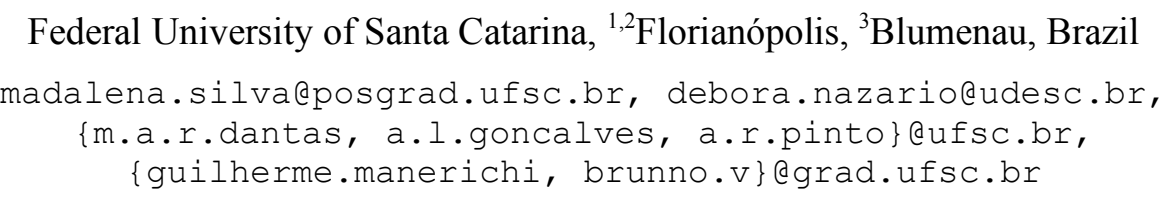

\begin{abstract}
Most research on Human Biometeorology use information from health and weather data providers to correlate and infer the impacts of weather variables on some morbidities. Although research generates indicators for the health management, it needs to be refined and consider weather variables in the indoor environment and provide these data for evaluation in conjunction with the patient's clinical data. In order to contribute to human biometeorology, this paper proposes implementing the IoT (Internet of Things) using the ZigBee to monitor the environmental conditions of a ubiquitous AAL (Ambient Assisted Living) setting, and to store data in the cloud in order to be consumed by caregiver health.
\end{abstract}

Resumo. A maioria das pesquisas sobre a Biometeorologia Humana usa dados de provedores de dados da saúde e do clima, para correlacionar e inferir os impactos das variáveis climáticas sobre as morbidades. Embora possam gerar indicadores para a gestão da saúde, as pesquisas precisam ser refinadas no sentido de considerar as leituras das variáveis meteorológicas no ambiente interno e, disponibilizar esses dados a serem avaliados juntamente com os dados clínicos dos pacientes. Visando contribuir com a Biometeorologia Humana, neste artigo é proposta a implementação da Internet das Coisas (IoT), com o uso da ZigBee, para monitorar as condições ambientais de um AAL (Ambient Assisted Living) ubíquo, e armazenar os dados na nuvem, para serem consumidos por cuidadores da sáude.

\section{Introdução}

A Biometeorologia Humana é a ciência que estuda o impacto da influência atmosférica sobre a saúde e bem estar do homem. São muitas as propostas existentes na literatura que correlacionam as condições climáticas com a saúde, incluindo o conforto térmico [Thom 1959], as morbidades [Quinn et. al., 2014], a mortalidade [Quinn et al., 2014, Zhang et al., 2014], a saúde fetal [Ngo e Horton, 2016], entre tantas outras. A grande maioria usa dados abertos de provedores de dados da saúde e do clima, para correlacionar e fazer inferências sobre os impactos das variáveis climáticas sobre algumas morbidades de um país, região ou grupo de pessoas [Critchfield 1983 e Azevedo et al., 2015].

Embora as pesquisas citadas possam gerar indicadores para a gestão da saúde, as mesmas precisam ser refinadas, uma vez que existe diferença na precisão dos valores das variáveis meteorológicas lidas no ambiente externo versus interno [Quinn et al., 2014]. Outro aspecto a considerar é a provisão desses dados, junto ao PEP (Prontuário Eletrônico do Paciente) para auxiliar nas análises médicas. 
Uma das morbidades que tem sido fortemente associada com alterações climáticas são as Infecções Respiratórias Agudas (IRA) [Gonçalves e Colho 2010, Araújo et al. 2010, Azevedo et al. 2015]. Como as pessoas vivem a maior parte do tempo em ambientes internos e muitas delas têm propensão a desencadear algum tipo de doença respiratória, torna-se extremamente recomendado a monitoração das condições meteorológicas no ambiente interno. Os valores das variáveis meteorológicas, associados ao contexto do utente (i.e. estado clínico, histórico do paciente/familiar) tornam-se indicadores valiosos para a tomada de decisões dos cuidadores da saúde.

Usando a tecnologia a serviço da Biometeorologia Humana, neste artigo é proposta a implementação da Internet das Coisas (IoT), com o uso das Redes de Sensores Sem Fio (RSSF) ZigBee, para monitorar as condições ambientais (temperatura do bulbo seco, temperatura do ponto de orvalho, umidade relativa do ar) de um AAL (Ambient Assisted Living) ubíquo, comunicando objetos físicos (sensores, dispositivos e máquinas) e armazenando dados provenientes destes na nuvem.

Para validar a proposta, foi idealizado um cenário de um paciente idoso, com pré disposição a desencadear crises asmáticas. O mesmo é assistido por cuidadores formais e informais da saúde. Nos experimentos, configuramos uma infraestrutura física para gerar, armazenar e consumir os dados persistidos na nuvem, com diferentes visões do sistema. Uma análise sobre os dados gerados foi realizada e o sistema demonstrou um percentual de $94 \%$ de confiabilidade.

O artigo está organizado em seções. Na seção 2 são apresentadas as pesquisas sobre o uso das RSSF e IoT em aplicações de Biometeorologia Humana. A seção 3 apresenta a proposta e a implementação do sistema. A seção 4 apresenta os experimentos e os resultados. Finalizando, a seção 5 apresenta as considerações finais.

\section{Revisão da Literatura}

A seção investiga o uso da RSSF aplicada na área da saúde, sobretudo a ZigBee com IoT. Também são apresentados alguns trabalhos que correlacionam fatores meteorológicos do ambiente interno a saúde das pessoas.

\subsection{Uso das Redes de Sensores Sem Fio na Biometeorologia Humana}

A Figura 1 sumariza a quantidade de publicações que usam RSSF na área da saúde. Dos 594 documentos publicados (2005 a 15 de Julho de 2015), a grande maioria utilizou a ZigBee para o monitoramento de sinais biomédicos, usando redes de sensores do corpo; monitoramento de atividades de idosos em AAL, entre outras. Destas, apenas 6 utilizaram a ZigBee para monitoramento de variáveis meteorológicas do ambiente interno [Yang et al. 2014a, Sun et al. 2013, Nam et al. 2011, Bader et al. 2009, Jayakumar et al. 2015, Sung et al. 2012]. Essa evidência motivou a escolha de usar e avaliar a confiabilidade da ZigBee num cenário de Biometeorologia Humana (seção 4).

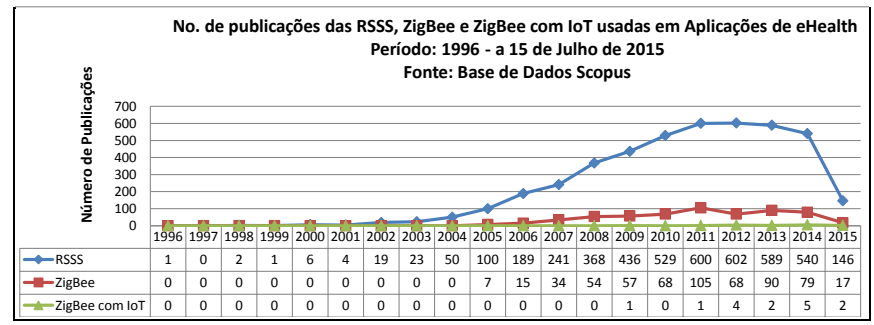

Figura 1. Número de publicações RSSF, RSSF e ZigBee, ZigBee em loT.

As 15 publicações que utilizaram a IoT com ZigBee, demonstram que a IoT e ZigBee é o conjunto de tecnologia mais adequado a ser usado no monitoramento de sinais biomédicos. $\mathrm{O}$ 
trabalho descrito em [Yang et al. 2014b] utiliza a IoT e ZigBee para estruturar um ambiente de monitoramento inteligente das condições físicas e qualidade do ar de um hospital.

\subsection{Correlações da Temperatura e Umidade Relativa do Ar com Crises Asmáticas}

Segundo os autores [Teixeira et al. 2012], desde os primórdios a umidade relativa (UR) do ar (alta ou baixa) tem sido relacionada a problemas de saúde, devendo ficar entre $40 \%$ a $70 \%$. Em seus estudos, os autores [Collins 1986] evidenciaram que tanto a UR baixa quanto a alta podem contribuir para o aparecimento de doenças respiratórias.

Segundo a OMS (Organização Mundial da Saúde), a asma é uma das doenças respiratórias que tem sido apontada como problema mundial de saúde pública. De acordo com GIMA os fatores que podem desencadear ou agravar os sintomas da doença incluem infecções virais, alérgicos domésticos ou profissionais (por exemplo, ácaros, pólens, barata), o fumo do tabaco, exercício e stress. Além destes, os parâmetros meteorológicos (i.e. umidade relativa do ar, precipitação, temperatura do ponto de orvalho, horas de sol e temperatura de bulbo seco) têm sido estudados como indicadores da frequência de asma brônquica [Fleischer 1978].

Outros estudos correlacionam às crises asmáticas com as variáveis, temperatura e umidade [Ayres Sampaio et al. 2014, Teixeira e Postolache 2014, Ezequiel et al. 2007, Santic' et al. 2002, Zaninovic e Raos 2001, Shimizu et al. 2001, Strannegard and Strannegard 1990, Ito et al. 1989, Fleischer 1978, Paulus e Smith 1967].

\subsection{Correlação da Temperatura do Ponto de Orvalho, como Indicador de Desconforto Térmico Humano}

O ponto de orvalho pode ser usado como indicador de desconforto térmico, fazendo-se uma relação com a temperatura, pressão e a umidade. O ponto de orvalho refere-se à temperatura na qual o vapor de água presente no ar ambiente passa ao estado líquido na forma de pequenas gotas por via da condensação. A Tabela 1 [Horstmeyer 2006] apresenta os níveis de sensação no ser humano, em função do cálculo da temperatura de orvalho, levando em consideração a UR a uma temperatura ambiente de $32^{\circ} \mathrm{C}$.

Tabela 1. Sensação Términa em função da Temperatura do Ponto de Orvalho

\begin{tabular}{c|l|c|c}
\hline $\begin{array}{c}\text { Ponto de } \\
\text { Orvalho em }\end{array}{ }^{\circ} \mathrm{C}$ & \multicolumn{1}{|c|}{ Sensação Térmica no ser Humano } & UR a 32 ${ }^{\circ} \mathbf{C}$ & $\begin{array}{c}\text { Sensação Térmica } \\
\text { dos 32 }\end{array}$ \\
\hline$>2$ com UR $^{\circ} \mathrm{C}$ & Extremamente sufocante. Casos de morte por hipertermina & $>=85 \%$ & $54^{\circ} \mathrm{C}-47^{\circ} \mathrm{C}$ \\
\hline $26-28^{\circ} \mathrm{C}$ & Altíssima. Mortal para doenças relacionadas à asma & $84-73 \%$ & $46^{\circ} \mathrm{C}-42^{\circ} \mathrm{C}$ \\
\hline $24-26^{\circ} \mathrm{C}$ & Extremamente desconfortável, consideravelmente sufocante & $72-62 \%$ & $41^{\circ} \mathrm{C}-38^{\circ} \mathrm{C}$ \\
\hline $21-24^{\circ} \mathrm{C}$ & Muito úmido, desconfortável & $61-52 \%$ & $37^{\circ} \mathrm{C}-35^{\circ} \mathrm{C}$ \\
\hline $18-21^{\circ} \mathrm{C}$ & Desconfortável para a maioria das pessoas no limite superior & $51-44 \%$ & $35^{\circ} \mathrm{C}-33^{\circ} \mathrm{C}$ \\
\hline $16-18^{\circ} \mathrm{C}$ & $\begin{array}{l}\text { Aceitável para a maioria, porém todos percebem a umidade no } \\
\text { limite superior }\end{array}$ & $43-37 \%$ & $32^{\circ} \mathrm{C}$ \\
\hline $13-16^{\circ} \mathrm{C}$ & Confortável & $36-31 \%$ & $32^{\circ} \mathrm{C}-31^{\circ} \mathrm{C}$ \\
\hline $10-12^{\circ} \mathrm{C}$ & Muito confortável & $30-26 \%$ & $31^{\circ} \mathrm{C}-30^{\circ} \mathrm{C}$ \\
\hline$<10^{\circ} \mathrm{C}$ & Um pouco seco para algumas pessoas & $<=25 \%$ & $30^{\circ} \mathrm{C}-27^{\circ} \mathrm{C}$ \\
\hline
\end{tabular}

A Tabela 2 apresenta as equações necessárias para obter o índice de desconforto térmico e demais parâmetros usados no sistema proposto (seção 3).

Tabela 2. Equações para Obtenção dos Valores das Variáveis Metereológicas

\begin{tabular}{|c|c|c|c|}
\hline Cálculo & Equações & Variáveis & Autores \\
\hline $\begin{array}{l}\text { Índice de Desconforto } \\
\text { Térmico } \\
\text { (ID) }\end{array}$ & $I D=0.99 \times \mathrm{Ta}+0.36 \times$ To +41.5 & $\begin{array}{l}\text { ID }=\text { índice de desconforto } \\
\mathrm{Ta}=\text { temperatura em graus }{ }^{\circ} \mathrm{C} \\
\text { To }=\text { temperatura de orvalho em }{ }^{\circ} \mathrm{C}\end{array}$ & $\begin{array}{l}\text { [Horstmeyer } \\
\text { 2006], adaptada de } \\
\text { [Thom 1959] }\end{array}$ \\
\hline $\begin{array}{l}\text { Temperatura do Ponto } \\
\text { de Orvalho } \\
(\mathrm{To}) \mathrm{em}^{\circ} \mathrm{C}\end{array}$ & $T o=\frac{B_{1} \times\left[\operatorname{Ln}\left(\frac{U r}{100}\right)+\left(\frac{A_{1} \times T a}{B_{1}+T a}\right)\right]}{A_{1}-\operatorname{Ln}\left(\frac{U r}{100}\right)-\left(\frac{A_{1} \times T a}{B_{1}+T a}\right)}$ & $\begin{array}{l}A 1=17,625 \\
B 1=243,04 \\
L n=\log 2,718 \\
U r=\text { umidade relativa }\end{array}$ & [Lawrence 2005] \\
\hline
\end{tabular}




\begin{tabular}{|c|c|c|c|}
\hline $\begin{array}{l}\text { Umidade Relativa } \\
\text { (UR) em \% }\end{array}$ & $U r=100 \times\left(\frac{e}{e_{s}}\right)$ & $\begin{array}{l}e=\text { pressão atual de vapor } \\
e s=\text { pressão saturada de vapor }\end{array}$ & [Lawrence 2005] \\
\hline $\begin{array}{l}\text { Pressão Saturada do } \\
\text { Vapor } \\
\text { (es) }\end{array}$ & $e_{S}=E \times 10^{\left(\frac{7,5 \times T a}{237,3+T a}\right)}$ & $\begin{array}{l}\mathrm{E}=\text { parâmetro equivalente a } 0,6108 \\
\text { para resultados em kilopascal }(\mathrm{kPa}) \\
\text { e } \mathrm{Ta} \text { em graus }{ }^{\circ} \mathrm{C} .\end{array}$ & [Lier et al. 2008] \\
\hline
\end{tabular}

\section{Implementação da IoT para Monitoramento das Condições Ambientais do AAL}

A Figura 2 apresenta uma visão geral da proposta. O sistema funciona da seguinte forma. Os dados lidos dos sensores biomédicos são enviados via conexão USB para o sistema de gerenciamento de contexto. Os dados capturados dos sensores de ambiente são enviados ao Gateway IoT e deste, são encaminhados para o sistema de gerenciamento de contexto, via comunicação wireless (802.11). No sistema de gerenciamento de contexto, ocorre a gestão da qualidade do contexto, ou seja, é realizado o filtro dos valores lidos e somente os valores válidos, acurados, precisos e atuais são enviados para a nuvem [Nazário et al., 2014]. Uma vez filtrados, os dados são enviados para o gateway doméstico usando conexão ethernet (802.3). O transporte dos dados para a nuvem é feito através de túneis criptografados, usando VPN (Virtual Private Network) e somente pessoas autorizadas (cuidadores formais, informais, grupos familiares e serviços de manutenção), com diferentes visões do sistema, podem acessar os dados.

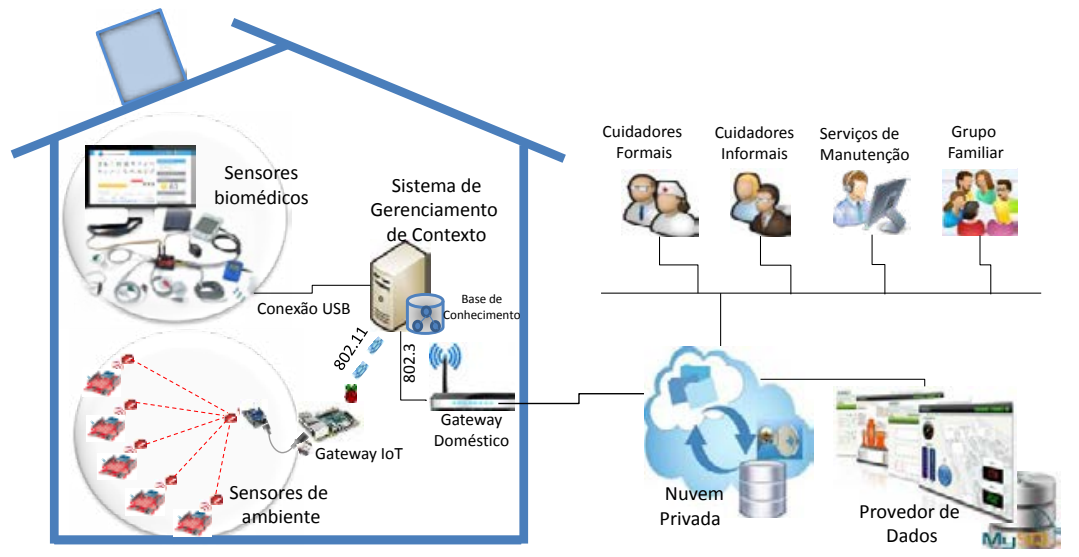

Figura 2. Visão Geral do Sistema Proposto

\subsection{Configuração dos Sensores Biomédicos e Distribuição do Contexto}

Inicialmente foi proposta uma arquitetura para o gerenciamento da QoC (Quality of Context) dos dados dos sensores biomédicos [Nazário et al., 2014] usando a plataforma eHealth [Cooking-hacks s.d.], num AAL ubíquo. Para a distribuição do contexto do AAL ubíquo (sinais biomédicos e dados do utente) para a nuvem, uma infraestrutura de transporte usando SDN (Software Defined Network) foi proposta e validada [Da Silva et al., 2015]. Portanto, já realizamos os experimentos e validações da captura, processamento e distribuição dos sinais biomédicos do AAL ubíquo para a nuvem. Agora, iremos nos concentrar em agregar a ZigBee com IoT para a monitoração das variáveis meteorológicas, armazenando-as numa base de dados na nuvem para serem consumidas por cuidadores da saúde.

\subsection{Configuração dos Sensores de Ambiente}

A rede ZigBee foi configurada com topologia em estrela, composta por 5 nodos escravos e 1 nodo controlador. Na topologia foram usados 5 módulos XBee Antenna 1Mw Serie 1. Cada módulo é conectado na Xbee shield, que por sua vez é encaixada na placa Arduino Uno. Em cada placa Arduino de um nodo escravo está plugado o sensor de temperatura e umidade, DHT11. 
O elemento sensor do DHT11 é um termistor do tipo NTC, capaz de fazer leituras de temperaturas entre 0 a $50^{\circ} \mathrm{C}$ com $\pm 2^{\circ} \mathrm{C}$ de precisão. $\mathrm{O}$ elemento sensor de umidade, do tipo HR20, é capaz de ler a umidade entre 20 a $90 \%$ com $\pm 5 \%$ de precisão.

O DHT11 possui um circuito interno programado para fazer a leitura dos sensores e enviar ao microcontrolador Arduino, via comunicação serial. $O$ Arduino envia um pulso negativo de $18 \mathrm{~ms}$ para o sensor DHT11, seguido de um pulso positivo de $20 \mu \mathrm{S}$ a $40 \mu \mathrm{S}$. Logo após, o DHT11 envia uma resposta para o Arduino, seguido dos bits de dados provenientes das medições. O sensor DHT11 envia 40 bits ( 5 bytes) mais os bits de inicialização.

\subsection{Implementação da IoT para a Comunicação entre os Dispositivos da RSSF ZigBee e Raspberry PI}

Para obter as leituras das medições, via troca de mensagens, entre o coordenador da rede e os nodos ZigBee foram programados dois códigos na linguagem $\mathrm{C}$, um para os nodos escravos $\mathrm{e}$ outro para o controlador. Para o nosso experimento foram usadas as bibliotecas XBee e DHT. A biblioteca XBee é responsável pela comunicação entre os nodos da rede ZigBee. A biblioteca DHT possui funções prontas para leitura e escrita dos dados da temperatura em graus Celsius e umidade em percentual.

\section{A. Leitura da Temperatura e Umidade pelos Nodos ZigBee}

Ao integrar a rede, cada nodo escravo recebe uma identificação e fica no aguardo das solicitações originadas pelo nodo controlador. Ao receber uma mensagem do controlador cujo ID seja o seu identificador, o nodo escravo coleta e envia a leitura dos dados do sensor ao controlador.

\section{B. Leitura de Temperatura e Umidade pelo Controlador ZigBee}

O controlador inicia o processo de comunicação, enviando requisições em sequência, para cada nodo da rede. As mensagens geradas pelo controlador contém o número de identificação do nodo e são enviadas a cada intervalo de tempo de $1500 \mathrm{~ms}$. As mensagens são enviadas sempre a cada $1500 \mathrm{~ms}$, independente do tempo da resposta recebida. Ao receber a resposta e realizar o cálculo do ponto do orvalho, o controlador coloca a mensagem no formato de endereço e envia para a porta de comunicação serial do Arduino, que está conectada diretamente na porta serial do Raspberry PI.

A biblioteca DHT não possui um método para cálculo do ponto de orvalho, no entanto, a partir dos valores lidos (temperatura e umidade) é possível criar um método para estimar este valor. Nós programamos o método "dewPoint", aplicando a Equação 2. As Equações 3 e 4, não foram necessárias, pois o método de escrita da umidade, nativo da biblioteca DHT, já fornece a umidade formatada em percentual.

\section{3) Implementação do Gateway IoT}

O Raspberry PI atua como gateway IoT. Ele recebe os dados de sensoriamento do coordenador ZigBee via comunicação serial. Os dados recebidos são processados no Raspberry PI e enviados para o sistema de gerenciamento do contexto. Após a etapa do filtro [Nazário et al., 2014], os dados são enviados para a nuvem usando a VPN. A transmissão dos dados do Raspberry PI para o sistema de gerenciamento de contexto ocorre via interface wireless, com o roteador wireless.

\subsection{Persistência dos Dados}

Para implementar a persistência de dados foi instalado o sistema supervisório ScadaBR num servidor Web, na nuvem. Os dados são enviados via protocolo HTTP e persistidos na base de dados MySql do sistema supervisório.

\section{A. Data Source e Data Points do AAL}


Para enviar os dados ao ScadaBR, foi criada uma fonte de dados do tipo HTTP. A fonte de dados recebe um nome, está associada a um endereço IP. No nosso experimento a fonte de dados representa um AAL e pode ter múltiplos usuários autorizados no seu gerenciamento. Para cada nodo da rede ZigBee foram criados três pontos de dados para armazenar os valores das variáveis temperatura ambiente, umidade e temperatura do ponto de orvalho. Cada ponto de dado está associado a um cômodo do AAL.

\section{B. Monitoramento dos Dados dos Sensores do AAL}

Para monitorar os pontos da fonte de dados foi utilizado o recurso watch list do ScadaBR. Esse recurso permite criar listas dinâmicas dos pontos com seus valores associados, visualizar os últimos tempos de atualização e gerar gráficos de informações históricas.

\section{Tratamento de Eventos}

Este recurso foi usado para gerar alertas por tratadores de eventos, sempre que os valores das variáveis monitoradas estejam fora ou próxima dos limites mínimos e máximos aceitáveis. Foi utilizado o alarme como tratador de eventos.

\section{Ambiente e Resultados Experimentais}

Nossos experimentos consistiram em gerar dados em tempo real de sinais biomédicos, juntamente com as variáveis meteorológicas do AAL, enviá-los para a nuvem e analisar o comportamento do sistema, no consumo dos dados pelo cuidador formal e informal e, avaliar a confiabilidade do sistema referente aos pacotes enviados em relação aos entregues.

\subsection{Cenário Experimental}

Idealizamos e criamos um cenário real, com todos os componentes e dispositivos descritos na seção 3. Para a realização dos experimentos, as três entidades (utente no AAL, provedor de dados e cuidador da saúde) foram distribuídas em diferentes cidades de um mesmo estado. $\mathrm{O}$ utente monitorado é um paciente idoso, capacitado e que possui histórico de crises asmáticas.

Dois usuários, com diferentes visões do sistema foram criados, representando, um cuidador formal e um cuidador informal da saúde. O cuidador informal está autorizado em visualizar a monitoração das condições ambientais do AAL, diretamente da IHC do ScadaBR. E o cuidador formal pode consumir a fonte de dados do AAL a partir de uma aplicação externa, pois, pode haver a necessidade de correlacionar os valores das variáveis meteorológicas monitoradas com os dados clínicos do utente.

Com a plataforma de eHealth [Cooking-hacks s.d.] foram usados os sensores de pulso, oxigenação no sangue, temperatura corporal, pressão sanguínea, posição e queda do paciente. $O$ tempo entre leituras de 5 minutos foi usado para os sensores de pulso, oxigênio, temperatura corporal e posição do paciente. E para a pressão arterial foi feita a leitura a cada 2 horas. Foram realizadas 9 baterias de testes, totalizando 807 leituras dos sensores biomédicos.

Para monitorar a temperatura ambiente, temperatura do ponto de orvalho e umidade relativa do ar, foram usados os sensores DHT11, com 5 nodos ZigBee e 1 nodo coordenador. Os nodos ZigBee, foram configurados para enviar amostras das leituras dos sensores DHT11 a cada 15 segundos. As monitorações foram avaliadas, por um período de 30 dias.

\subsection{Monitoração das Variáveis Meteorológicas do AAL pelo Cuidador Informal}

O cuidador informal é um usuário autorizado para monitorar a temperatura, umidade relativa e ponto de orvalho em qualquer cômodo do AAL e tomar algumas ações de sua competência quando observar anormalidades. Alguns tratadores de eventos foram configurados para gerar alertas em situações anormais ou que exigem atenção. 
No caso da temperatura, foram usados os detectores de eventos para limites superior a $32^{\circ} \mathrm{C}$ e inferior a $10^{\circ} \mathrm{C}$. No período monitorado as temperaturas medidas ficaram dentro dos limites estabelecidos (Figura 3). O ScadaBR foi configurado para armazenar os valores da temperatura apenas quando o valor da mesma sofrer alteração.

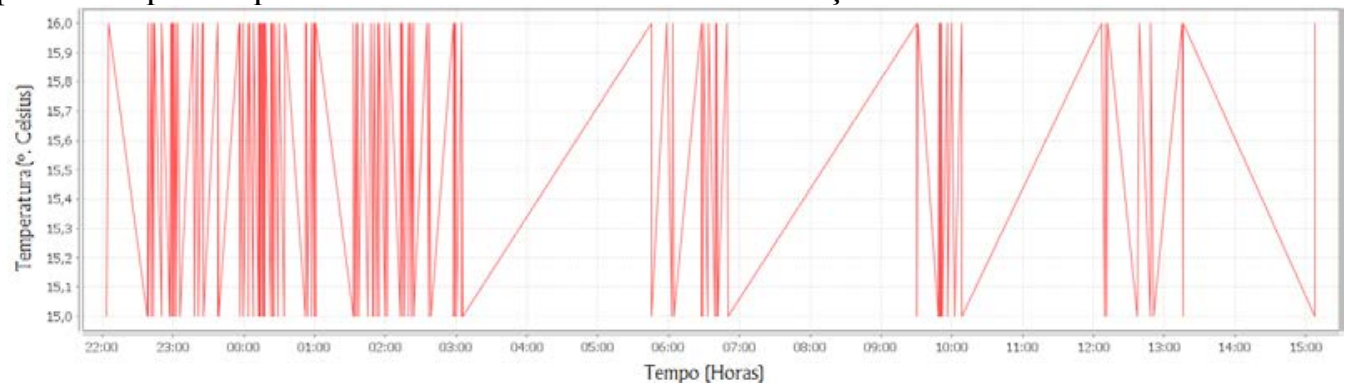

Figura 3. Temperatura Ambiente em um dos Cômodos do AAL

A OMS preconiza que os valores ideais da umidade relativa devem ficar na faixa de $40 \%$ a $70 \%$. Sendo que de $20 \%$ a $30 \%$ exige-se atenção e alguns cuidados; de $12 \%$ a $20 \%$ devese entrar em estado de alerta e abaixo de $12 \%$ a situação torna-se emergencial. Os detectores de alertas e níveis de alarmes para umidade relativa foram definidos com base nessas recomendações. No período das monitorações, a umidade relativa ficou entre 48 a 51\% (Figura 4).

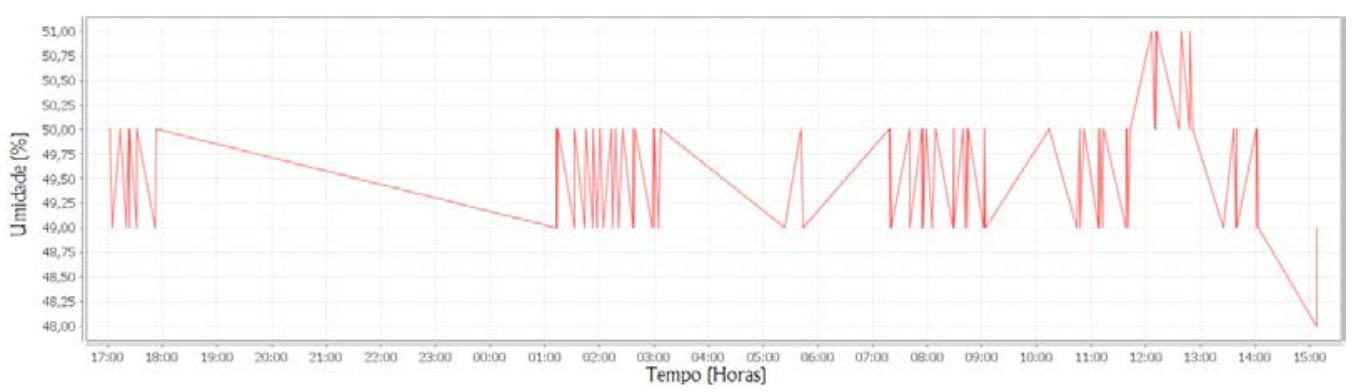

Fugura 4. Umidade Relativa em um dos Cômodos do AAL

\subsection{Monitoração do AAL pelo Cuidador Formal}

O cuidador formal realiza as análises das medições diretamente no PEP (Prontuário Eletrônico do Paciente), pois este necessita avaliar os dados clínicos do utente, o histórico das crises asmáticas e se for o caso correlacionar os fatores meteorológicos, com as crises asmáticas, fazer inferências e descobrir novos fatos.

Note que nosso objetivo não consiste em programar um PEP e sim, disponibilizar essas informações para serem consumidas por uma aplicação de terceiros e avaliar como o sistema proposto se comporta. Por outro lado, o fato das informações do AAL estarem disponíveis na mesma aplicação facilita e pode agilizar a avaliação do cuidador. Para isso prototipamos uma aplicação em Java (Figura 5), capaz de ler as informações do AAL, persistidas no MySql do ScadaBR. Opcionalmente, o cuidador formal, pode salvar no PEP a data, a hora e os valores das variáveis monitoradas, para fazer o registro, juntamente com a ocorrência da crise asmática.

Na Figura 5 pode-se observar que a monitoração das condições ambientais do AAL pode ser realizada por cômodo, por período de tempo e pelo filtro de valores e operadores relacionais para buscar a temperatura e a umidade. Observa-se também que a aplicação exibe o índice de conforto térmico, de acordo com os valores medidos, ou seja, a uma temperatura de $27^{\circ} \mathrm{C}$, com umidade relativa a $41 \%$ e temperatura do ponto de orvalho de $12^{\circ} \mathrm{C}$, a sensação de conforto para o utente é "muito confortável". 


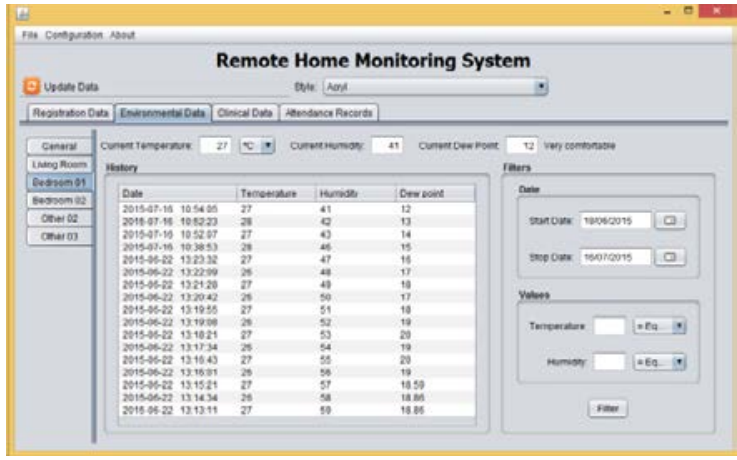

Figura 5. Monitoramento Remoto Usada pelo Cuidador Formal

Para o cálculo do índice de conforto, foi utilizada a Equação 1. A mesma foi calculada, com os valores existentes na base de dados do ScadBR. Para o cálculo, além do uso da temperatura e umidade, usou-se o valor da temperatura do ponto de orvalho (Equação 2).

\subsection{Confiabilidade do Sistema Proposto}

Para determinar a confiabilidade do sistema, os dados dos sensores de ambiente foram monitorados por um período de um mês. Um registro de $\log$ foi gerado e analisado no Raspberry PI para manter informações de pacotes recebidos com sucesso dos nodos ZigBee. O log mantém o ID do nodo, o tempo e o contador de pacotes. E com isso, foi possível obter o percentual de confiabilidade no envio dos dados dos nodos ZigBee para o Gateway IoT, conforme ilustrado na Tabela 3.

Tabela 3. Confiabilidade do Sistema em Função da Entrega dos Pacotes

\begin{tabular}{c|c|c|c|c|c}
\hline $\begin{array}{c}\text { ID do } \\
\text { nodo }\end{array}$ & $\begin{array}{c}\text { Tempo médio } \\
\text { entre chegadas }\end{array}$ & $\begin{array}{c}\text { Número de pacotes } \\
\text { esperados }\end{array}$ & $\begin{array}{c}\text { Número de pacotes } \\
\text { recebidos }\end{array}$ & $\begin{array}{c}\text { Número de } \\
\text { pacotes perdidos }\end{array}$ & $\begin{array}{c}\text { Confiabilidade } \\
(\%)\end{array}$ \\
\hline 1 & 15,2 & 176211 & 166791 & 9420 & 94,65412186 \\
\hline 2 & 15,8 & 169519 & 159619 & 9900 & 94,15994624 \\
\hline 3 & 16,2 & 165333 & 153993 & 11340 & 93,14112903 \\
\hline 4 & 15,6 & 171692 & 161896 & 9796 & 94,29440508 \\
\hline 5 & 15,5 & 172800 & 163006 & 9794 & 94,33224537 \\
\hline
\end{tabular}

Para o envio dos dados do Gateway IoT para o ScadaBR, usou-se o protocolo de transporte TCP, neste caso, o próprio protocolo possui mecanismos de controle de erro, garantindo confiabilidade entre origem e destino.

\section{Considerações e Continuidade do Trabalho}

Através das pesquisas evidenciamos que as variáveis meteorológicas têm sido usadas para avaliar a qualidade do ar, no ambiente interno, bem como fazer correlações com crises asmáticas. Embora, a ZigBee [Yang et al. 2014b] seja considerada bastante flexível e mais adaptável às RSSF, observamos que existem pouquíssimas propostas de IoT com ZigBee na área da saúde. Isso demonstra que a aplicabilidade da IoT e ZigBee na aquisição e transmissão de sinais em AAL ubíquo, é carente de investigação.

Evidenciando a necessidade descrita acima, foi proposto e implementado a IoT com a RSSF ZigBee para o monitoramento das condições do ar ambiente de um AAL, visando os cuidados do utente com alguma pré-disposição a crises asmáticas. Para validar nossa proposta realizamos os experimentos, com as três principais entidades (utente no AAL, provedor de dados e cuidador da saúde) situadas em diferentes cidades. Os resultados foram satisfatórios, uma vez que foi possível gerar gráficos e alertas em tempo quase real; disponibilizar os dados das variáveis climáticas do AAL tanto no IHC do sistema supervisório, quanto para serem consumidos por uma aplicação externa. Além disso, o sistema demonstrou uma confiabilidade de $94 \%$. Contudo, convém implementar a tolerância a falhas para melhorar este percentual. 
Como propostas futuras, pretende-se: - configurar a ZigBee tolerante a falhas; escalar o número de AAL; migrar para uma base de dados NoSQL e criar um motor semântico para suportar a análise humana pelo cuidador da saúde.

\section{Referências}

Araújo, R. A. F., Gomes Filho, M. F. and Dantas, R.T. "Morbidades em Função das Variáveis Meteorologicas em Campina Grande - PB", In: Jacques Servain; José Nilson B. Campos; Eduardo Savio P.R. Martins; Dirceu Silveira Reis Junior. (Org.). Climat de'l Atlantique Tropical et Impacts sur le Nordeste (CATIN). 01 ed. Fortaleza - CE: Gráfica e Editora Ronda Ltda, 2010.

Alduchov, O. A. and Eskridge, R. E. (1996) "Improved Magnus form approximation of saturation vapor pressure". Journal of Applied Meteorology, v. 35, n. 4, p. 601-609.

Azevedo, J. V. V., Santos, A. C., Alves, T. L. B., Azevedo, P. V. and Olinda, R. A. (2015) "Influência do Clima na Incidência de Infecção Respiratória Aguda em Crianças nos Municípios de Campina Grande e Monteiro, Paraíba, Brasil”, Revista Brasileira de Meteorologia, v. 30, n. 4, p. 467-477.

Ayres-Sampaio, D., Teodoro, A.C., Sillero, N., Santos, C., Fonseca and J., Freitas, A. (2014) "An investigation of the environmental determinants of asthma hospitalizations: An applied spatial approach", Geography, v. 47, p. 10-19, ISSN 0143-6228.

Bader, E., Al-Shamma'a, A. and Shaw, A. (2009) "Design and implementation of low-power and wireless multi-sensor devices for industrial applications", Developments in eSystems Engineering (DESE), Second International Conference on, Abu Dhabi, p. 418-424.

Collins, KJ. (1986) "Low indoor temperatures and morbidity in the elderly", Oxford Journals Medicine Age and Ageing, v. 15, p. 212-220.

Cooking-hacks. e-health sensor platform v2.0 for arduino and raspberry pi [biometric/medical applications].

Critchfield, H. J. General Climatology. Englewood Cliffs: Prentice-Hall, 1983.

Nazário, D.C, Tromel, I.V.B., Dantas M.A.R., and Todesco, J.L. (2014) "Toward Assessing Quality of Context Parameters in a Ubiquitous Assisted Environment," IEEE Symposium on Computers and Communications - ISCC, p.1-6.

Da Silva, M.P., Dantas, M.A.R., Gonçalves, A.L., Pinto, A.R. (2015) "A managing QoE approach for provisioning user experience aware services using SDN," 11th ACM Symposium on QoS and Security for Wireless and Mobile Networks (Q2SWinet), p. 51-58.

Ezequiel, O.D.S., Gazeta, G.S. and Freire, N.M.D.S (2007) "Prevalência dos atendimentos por crises de asma nos serviços públicos do Município de Juiz de Fora (MG)”, J. bras. pneumol. v. 33, n.1.

Gonçalves, F. L. T. and Coelho, M. S. Z. S. (2010) "Variação da morbidade de doenças respiratórias em função da variação da temperatura entre os meses de abril e maio em São Paulo", Ciência e Natura, UFSM, 32 (1): 103-118.

Horstmeyer, S. (2006). Relative Humidity Relative to What? The Dew Point Temperature a better approach, in http://www.shorstmeyer.com/wxfaqs/humidity/humidity.html. Access in July 2015.

Ito, S., Kondo, H., Kawaoi, T., Hiruma, F., Takashima, H., Kim, B., Togasaki, K., Togo, T. and Abe, T. (1989) "Outbreaks of asthma attacks and meteorological parameters-multivariate analysis", Japanese Journal of Allergology.

Jayakumar, D., Omana, J., Sivakumar and M. and Senthil, B. (2015) "A safe guard system for mine workers using wireless sensor networks", International Journal of Applied Engineering Research 10(8):21429-21441.

Fleischer, S.L.M. and Asnani, G.C (1978) "The influence of weather on asthma in Nairobi", International Journal of Biometeorology, v. 22, p. 263-270.

GIMA, Global Initiative for Asthma. Available in http://www.ginasthma.org/. Access in July 2015. 
Lawrence, M. G. (2005) “The Relationship between Relative Humidity and the Dewpoint Temperature in Moist Air", American Meteorological Society, [s.1].

Lier, Q. D. V., Dam, J. C. V., Metselaar, K., Jong R. and Duijnisveld, W. H. M. (2008) "Macroscopic root water uptake distribution using a matric flux potential approach", Vadose Zone Journal, v.7, n.3, p.1065-1078.

Nam, J.-W., Kim, H.-T., Min, B.-B., Kim, K.-H., Kim, G.-S. and Kim, J.-C. (2011) "Ventilation control of subway station using USN environmental sensor monitoring system", International Conference on Control, Automation and Systems, pp.305-308.

Ngo, N. S. and Horton, R. M. (2016) "Climate change and fetal health: The impacts of exposure to extreme temperatures in New York City”, Environmental Research, v. 144, Part A, p. 158-164.

Paulus, H.J. and Smith, T.J. "Association of allergic bronchial asthma with certain air pollutants and weather parameters" (1967) International Journal of Biometeorology, v. 11, p. 119-127.

Quinn, A., Tamerius, J. D., Perzanowski, M., Jacobson, J. S., Goldstein, I., Acosta, L. and Shaman, J. (2014) "Predicting indoor heat exposure risk during extreme heat events", Science of The Total Environment, v. 490, p. 686-693.

Santić, Z., Santić, Z., Santić, K., Kondza, D. and Bogut, S. (2002) "The relationships between the asthma and weather", Medicinski Arhiv, v. 56, n. 3, p. 155-157.

SCADABR. Available in http://www.scadabr.com.br/. Access in May 2015.

Sun, F.M., Fang, Z., Zhao, Z., Xu, Z.H., Tan, J., Chen, D.L., Du, L.D., Qian, Y.M., Hui, H.Y. and Tian, L.L. (2013) "A wireless ZigBee router with PHT sensing for health monitoring”, IEEE International Conference on Green Computing and Communications and IEEE Internet of Things and IEEE Cyber, Physical and Social Computing, GreenCom-iThings-CPSCom, pp. 1773-1778.

Shimizu, S., Kagawa, J. and Ishiguro, M. (2001) "The association between emergency clinic visits for asthmatic attacks and fluctuating environmental factors", Japanese Journal of Allergology, v. 50, n. 7, p.612-620.

Strannegard, I.-L. and Strannegard, O. (1990) "Childhood bronchial asthma in a desert country", Allergy: European Journal of Allergy and Clinical Immunology, v. 45, n. 5, p. 327-33.

Sung, W.-T., Chen, J.-H. and Wang, H.-C. (2012) "Wisdom health care environment systems for monitoring and automated control via RBF function", Applied Mechanics and Materials, v. 157-158, p. 315-318.

Teixeira G., F.L., Spohr Nedel, A. and Cardoso Alves, M.R (2012) "An analysis of the air relative humidity in internal and external environment in the city of São Paulo", Brazil, Revista Brasileira de Medicina, v. 69, n. 7.

Teixeira, F. A. and Postolache, O. (2014) "Wireless sensor network and web based information system for asthma trigger factors monitoring", Conference Record - IEEE Instrumentation and Measurement Technology Conference, pp. 1388-1393.

Thom EC (1959) “The discomfort index", Weatherwise, v. 12, p. 57-61.

Zhang, K., Li Y., Schwartz, J. D. and O'Neill, M. S. (2014) "What weather variables are important in predicting heat-related mortality? A new application of statistical learning methods", Environmental Research, v. 132, p. 350-359.

Yang, C.-T., Liao, C.-J., Liu, J.-C., Den, W., Chou, Y.-C. and Tsai, J.-J. (2014a) "Construction and application of an intelligent air quality monitoring system for healthcare environment", Journal of Medical Systems, v. 38, n. 2, p. 1-10.

Yang, C.-T., Liu, J.-C., Liao, C.-J., Wu, C.-C. and Le, F.-Y. (2014b) "On construction of an intelligent environmental monitoring system for healthcare", Parallel and Distributed Computing, Applications and Technologies, PDCAT Proceedings, art. no. 6904262, p. 246-253.

Zaninović, K. and Raos, M. (2001) "The influence of meteorological parameters on asthmatic attacks in children", Hrvatski Meteoroloski Casopis, v. 37, p. 89-94. 